\title{
FOCUSING SOLENOID FOR THE FRONT END OF A LINEAR RF ACCELERATOR*
}

\author{
I. Terechkine, V.V. Kashikhin, T. Page, M. Tartaglia, J. Tompkins \\ FNAL, Batavia, IL 60510, U.S.A.
}

\begin{abstract}
A prototype of a superconducting focusing solenoid for use in an RF linac has been built and tested at Fermi National Accelerator Laboratory (FNAL). The solenoid is comprised of the main coil, two bucking coils, two dipole corrector windings, and a low carbon steel flux return. At the excitation current of $250 \mathrm{~A}$, the magnetic field reaches $7.2 \mathrm{~T}$ in the center of the solenoid and is less than $5 \mathrm{G}$ on the axis at a distance of $150 \mathrm{~mm}$ from the center. The length of the solenoid is $150 \mathrm{~mm}$; the length of a cryovessel for the solenoid with a $20 \mathrm{~mm}$ diameter "warm" bore is $270 \mathrm{~mm}$. This paper presents the main design features of the focusing solenoid and discusses results from tests of the solenoid.
\end{abstract}

\section{INTRODUCTION}

To limit emittance growth in the low energy section of a high current linear ion accelerator, it is desirable to use an axially symmetric focusing system with smooth focusing and a relatively short focusing period. This approach promises a lower particle loss rate than can be achieved by using pairs of quadrupoles to focus the beam sequentially in the horizontal and vertical planes [1]. One way to implement this method is to employ superconducting solenoids as focusing elements. A high magnetic field achievable in superconducting solenoids helps to minimize the overall length along the beamline occupied by the focusing elements.

Such devices have been introduced in the design of a high power RF linac "front end" currently being studied at FNAL [2]. The front end consists of a radio-frequency quadrupole (RFQ) accelerating section followed by a normal conducting drift tube linac (DTL) structure and then a superconducting RF accelerating structure. A major requirement for focusing elements in the superconducting section of the linac is to limit the magnetic field on the accelerating cavity walls to the level of $\sim 1 \mu \mathrm{T}$. Although in the room temperature DTL section this requirement is relaxed to the level of $\sim 1 \mathrm{mT}$, it is still difficult to meet it while also maintaining the required short focusing period.

A study made to understand the problem and find a way to build the focusing solenoid resulted in the design algorithm described in [3]. To check different aspects of the chosen design approach, several prototype solenoids were built and tested. It was necessary to use high quality NbTi strand and achieve high winding density to meet the requirements for the solenoid strength and length. Some additional constraints also affected the design and fabrication.

*Work supported by US DOE under contract No. DE-AC02-07CH11359
To lower the fringe field, the solenoid was equipped with two bucking coils (BC) and a soft steel flux return. The magnetic field generated by the BC's oppose that of the main coil (MC), so an increase in the MC field strength is needed to bring the net focusing strength to the desired level.

About one third of the solenoids in the front end are equipped with pairs of corrector dipole (CD) windings for beam steering. The presence of CD's, wound on cylindrical surfaces just outside the beam pipe, leads to an increased inner diameter of the solenoid coils. This makes solving the fringe field issue more difficult.

In the room temperature DTL section, each lens is placed in its own cryostat, which uses additional space along the beam line. The restriction on longitudinal space further constrains the system design.

Prototypes of the focusing solenoids with and without correctors were built and tested. In this paper we present a description of the main design features and first test results; emphasis is given to one device with embedded dipole correctors.

\section{DESIGN AND FABRICATIOIN}

The schematic view in Fig. 1 shows one quarter of the longitudinal cross-section of a focusing solenoid with CD windings.

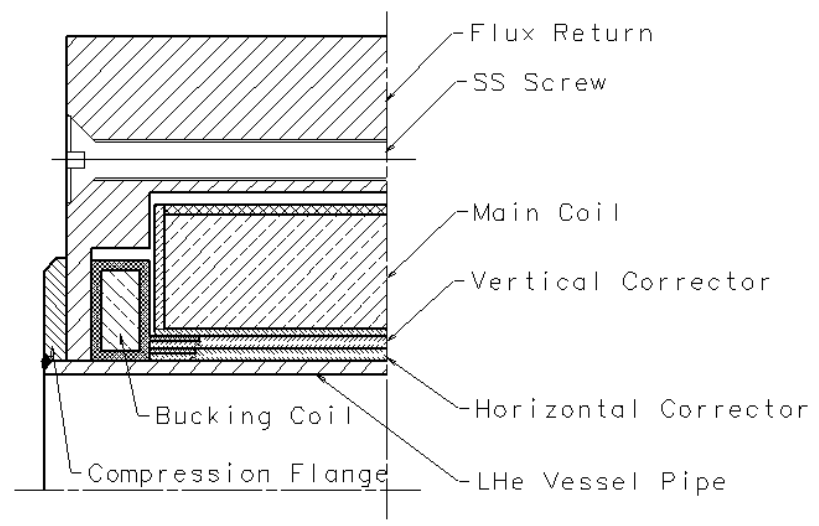

Figure 1: Design scheme of a focusing solenoid.

A stainless steel pipe is used as the inner wall of the liquid helium (LHe) vessel and serves as a base for the assembly. A cylindrical thick wall copper tube is slid above the LHe pipe. Evenly distributed along the perimeter of the tube are 36 holes where turns of the vertical and the horizontal dipoles are positioned. Insulated $\mathrm{NbTi}$ strand ( $0.8 \mathrm{~mm}$ bare diameter) was used to make the winding. The tube is placed inside a bobbin of the main coil, which is wound using similar NbTi strand and impregnated with epoxy. 
Epoxy impregnation is an important fabrication step which ensures the mechanical strength of the coils and allows the application of the pre-stress needed to limit internal motion in the solenoid and deformations of the coils during excitation. The impregnation also allows a simpler mechanical design of the coils' bobbins, and, most importantly, it significantly increases the thermal conductivity in the coils. This increase results in a higher threshold for thermal damage during quenching.

The bucking coils are wound using round $0.6 \mathrm{~mm}$ (bare diameter) NbTi strand; they are also impregnated with epoxy before being added to the assembly.

A low carbon steel flux return captures stray flux and is used as part of a mechanical structure which keeps the three coils in the assembly under compression. Stretched stainless steel rods in the flux return body and compression flanges welded to the pre-stretched LHe vessel pipe keep the two halves of the flux return together during the solenoid excitation, balancing an axial force of about eight metric tons.

Shown in the table below are winding data for the focusing solenoid with CD's.

Table 1: Winding Data

\begin{tabular}{|l|l|l|l|}
\hline Parameter & MC & BC & CD \\
\hline Strand dia (mm) & 0.8 & 0.6 & 08 \\
\hline ID (mm) & 55.2 & 53.2 & 50 \\
\hline OD (mm) & 97.5 & 98 & 53 \\
\hline Length (mm) & 74 & 6.5 & 75 \\
\hline Turns & 2234 & 400 & 16 \\
\hline
\end{tabular}

The assembled focusing solenoid is tested using a cold test Dewar system [4] before being welded into a stainless steel LHe vessel and positioned in a cryostat to form a lens assembly.

Each CD-equipped lens requires three pairs of leads. To reduce LHe loss, high temperature superconductor (HTS) leads will be used. Prototypes HTS lead tests are in preparation. A cross sectional view of an assembled lens is shown in Fig. 2.

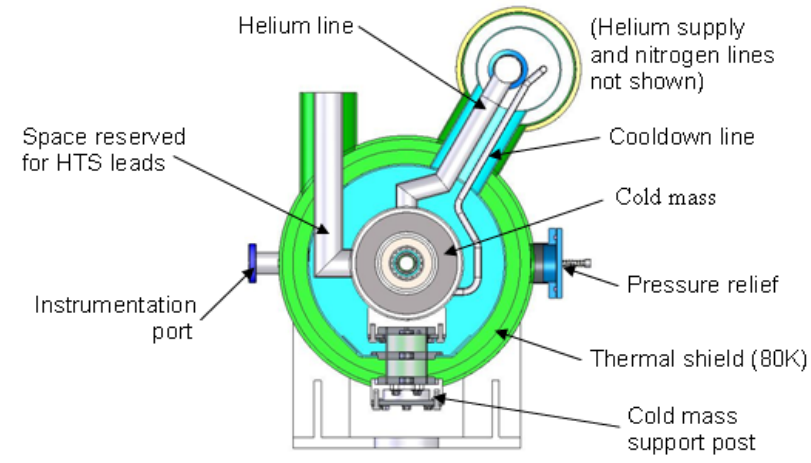

Figure 2: Cross-section view of an assembled lens.

The total (flange-to-flange) length of the assembled focusing lens is $270 \mathrm{~mm}$ while the distance from the center of the lens to the center of the nearest accelerating cavity is $\sim 260 \mathrm{~mm}$; the focusing period in this (DTL) section is $520 \mathrm{~mm}$.

\section{QUENCH PROTECTION}

The transition from superconducting to normal state in the current-carrying strand results in deposition of energy inside the strand and subsequent propagation of the normal zone (quenching). This can lead to an unacceptably high temperature rise inside the coil. Since each focusing solenoid of the DTL section of the linac stores an energy of $\sim 10 \mathrm{~kJ}$, it was important to learn whether this energy could damage or cause degradation of the solenoid. The effect of a quench depends on the current and the location in the solenoid at which the quench starts. The first evaluations of the effect gave encouraging results [3], but the absence of convenient and readily available means of making a comprehensive study of quench-related effects forced us to develop a specialized MATLAB-based tool [5] that allowed visualization of temperature and voltage to ground at any point inside the solenoid as a function of time. A provision for modeling a dump resistor was also included in the program. Using this software, a study of quench-related effects in a DTL solenoid confirmed that the most serious situation should be expected when quench starts in one of the bucking coils [6]. In this case, all the energy stored in the solenoid dissipates in the quenching coil; this results in a significant, although not dangerous, temperature rise. Figure 3 shows the temperature distribution after all the energy is dissipated inside the quenching BC in the "worst case" quench scenario: a 250 A excitation current (close to the quench limit), and the quench initiation point in the area of minimum magnetic field (turn \#1 and layer \# 20 in Fig. 3). A dump resistor was not used in this case and the protection system zeroed the power source immediately after quench detection. The maximum voltage to ground reached $\sim 500 \mathrm{~V}$ in the outer layer of the coil $\sim 80 \mathrm{~ms}$ after quench initiation.

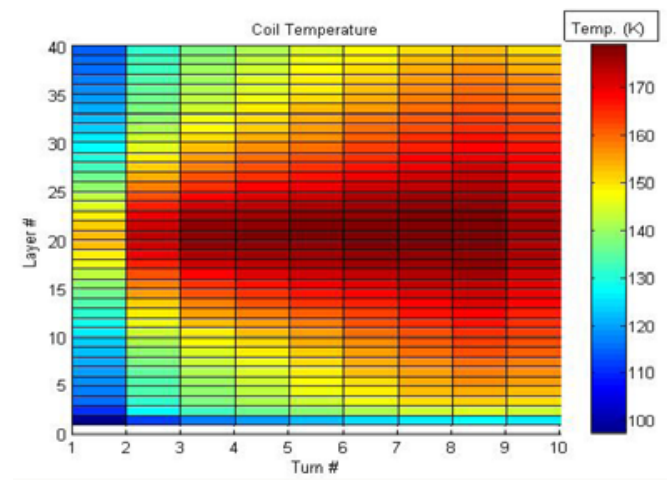

Figure 3: Temperature profile in the bucking coil

If a non-zero dump resistor is used, part of the energy stored in the system dissipates outside of the cryostat thus lowering the maximum temperature. The maximum voltage in this case depends on the discharge circuit: if it is configured as in Fig. 4 with BC1 quenching and with the outer layer of the MC grounded through a $1 \mathrm{Ohm}$ 
dump resistor, the maximum voltage to ground reaches $330 \mathrm{~V}$ and the maximum temperature is $\sim 90 \mathrm{~K}$ [7].

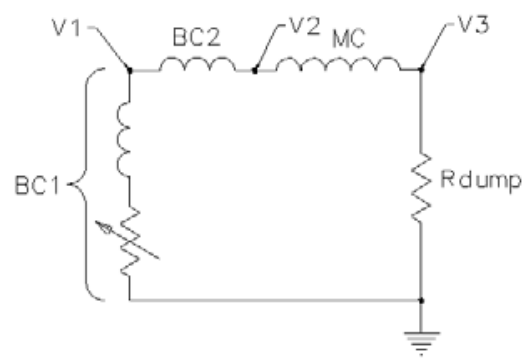

Figure 4: Solenoid discharge circuit.

\section{TEST RESULTS}

The first two focusing solenoids were extensively tested at $4.3 \mathrm{~K}$, and both showed rather short training histories. One solenoid, without embedded dipole correctors, was tested before and after welding into the LHe vessel and reached the predicted short sample limit (with slight retraining after the welding) of $255 \mathrm{~A}$ [8]. The second solenoid, with corrector dipoles, reached the maximum power supply current of $260 \mathrm{~A}$, just below the $270 \mathrm{~A}$ expected short sample limit [9]. The nominal focusing strength of this solenoid, $1.8 \mathrm{~T}^{2}$-m is reached at $\sim 230 \mathrm{~A}$, resulting in a margin of $\sim 13 \%$. A 0.6 Ohm dump resistor was used during the tests, but the coils survived quenching at the maximum current in one of the bucking coils even when the resistor was not connected.

During testing, significant attention was devoted to understanding details of the fringe magnetic field. Fig. 5 shows the measured profile of the magnetic field along the axis outside the solenoid, compared with the predicted fringe field.

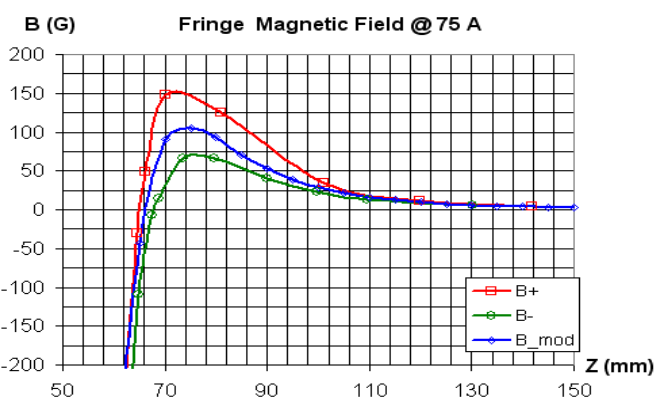

Figure 5: Fringe magnetic field at $75 \mathrm{~A}$

Due to differences in the winding parameters of the two bucking coils, deviating from the nominal parameters, the magnetic field outside the solenoid was not completely symmetrical. The fringe field evaluation was based on the nominal set of the winding parameters.

With the current in the solenoid of $250 \mathrm{~A}$, the current in the correctors could be set at the level of $275 \mathrm{~A}$. The measured profile of the transverse magnetic field can be approximated by the analytical expression below:

$$
\mathrm{B}(\mathrm{z}) / \mathrm{I}=-1.1810^{-4}+2.5110^{-8} \mathrm{z}+2.7310^{-8} \mathrm{z}^{2} \text {, }
$$

where $\mathrm{B}$ is in Tesla and $\mathrm{z}$ is in millimeters. With the current in the CD's of $250 \mathrm{~A}$, integration of this expression gives a transverse field integral of $0.26 \mathrm{~T}-\mathrm{cm}$, compared with the required $0.25 \mathrm{~T}-\mathrm{cm}$. So, at the maximum current, one expects a current margin of $\sim 4 \%$. This low margin was one of the reasons that forced us to modify the design of CD's. Tests of a modified version of the focusing solenoid with updated design of CD's are in progress.

\section{SUMMARY}

- A superconducting focusing solenoid has been developed for use in a DTL-type accelerating section of a high current proton (or $\mathrm{H}^{-}$) $\mathrm{RF}$ linac.

- The prototype solenoids provide the needed focusing strength, fit in the available space, and meet low fringe field requirement.

- The fabrication techniques used ensure quick training and reliable and reproducible performance.

- These DTL solenoids appear to be self protected in case of quench.

\section{ACKNOWLEDGEMENT}

Authors say special thanks to D. Dietderich of LBNL for his efforts to provide this R\&D work with quality NbTi strand developed for superconducting magnets of SSC. Authors are grateful to E. Barzi and D. Turrioni for testing the strand.

\section{REFERENCES}

[1] A. N. Mirzoian, P. N. Ostroumov, G. V. Romanov, and A. P. Fateev, "Increase of injection efficiency in a high current linear proton accelerator," Journal of Technical Physics, v. 25, pp. 710-713, 1980.

[2] P. N. Ostroumov, K. W. Shepard, G. W. Foster, I. V. Gonin, and G. V. Romanov, "Front end design of a multi-GeV H-minus linac,” PAC-05, Proceedings, pp. 3286-3288, 2006.

[3] G. Davis, V.V. Kashikhin, T. Page, I. Terechkine, J. Tompkins, and T. Wokas, "Designing focusing solenoids for superconducting RF accelerators," ASC-06, Seattle, 2006.

[4] R. Carcagno, et al, "Superconducting Solenoid Magnet Test Results,” ASC-06, Seattle, 2006.

[5] S. Obraztsov, I. Terechkine, "A Tool for Modeling Quench Propagation in Superconducting Focusing Solenoids and Related Protection Issues," TD-06063, FNAL, 2006.

[6] I. Terechkine, "CH Section Focusing Solenoid Quench Analysis,” TD-06-067, FNAL, 2006.

[7] I. Terechkine, "Using a Dump Resistor for Protection of Focusing Solenoids in the CH Section of the HINS Linac Front End,” TD-07-003, FNAL, 2007.

[8] C. Hess, et.al, "Focusing Solenoid CH_SOL_01, Fabrication Notes and Test Results," TD-07-006, FNAL, 2007.

[9] C. Hess, et.al, "Focusing Solenoid CH_SOL_02, Fabrication Notes and Test Results," TD-07-008, FNAL, 2007. 